\title{
VALENCY OF SELECTED RARE-EARTH IONS IN SnTe MATRIX
}

\author{
Z. GolACKI \\ Institute of Physics, Polish Academy of Sciences \\ Al. Lotników 32/46, 02-668 Warsaw, Poland \\ AND M. Heinonen \\ Department of Applied Physics, University of Turku, ElectroCity \\ Tykistokatu 2D, 20520 Turku, Finland
}

$\mathrm{X}$-ray photoelectron spectroscopy experiments on $\mathrm{SnTe}$ crystals doped by $\mathrm{Sm}, \mathrm{Eu}$, and $\mathrm{Yb}$ were performed to determine the valency of these ions in the $\mathrm{SnTe}$ matrix. The analysis of the X-ray photoelectron spectroscopy valence-band spectra revealed that $\mathrm{Eu}$ and $\mathrm{Yb}$ are divalent while $\mathrm{Sm}$ is trivalent in SnTe. These conclusions were confirmed by studies of the binding energies of core levels (4d-levels of $\mathrm{Yb}$ and $3 d$-levels of $\mathrm{Sm}$ and $\mathrm{Eu}$ ), which are not presented here.

PACS numbers: 79.60.-i

The rare-earth ( $\mathrm{RE}$ ) ions $\mathrm{Sm}, \mathrm{Eu}, \mathrm{Tm}$, and $\mathrm{Yb}$ can exist as divalent, trivalent or mixed valent ions in the solid state. The valency depends on chemical environment, lattice structure, and physical conditions such as pressure and temperature. Rare-earth monochalcogenides crystalize in the rock-salt structure, like SnTe and lead chalcogenides, forming mixed solid solutions with the latter. This provides us with many possibilities to investigate the influence of the environment on the valency of these ions, e.g., we can choose the first neighbors as Te, Se, and $\mathrm{S}$. On the other hand, we can keep the same anion, e.g., Te, and vary the lattice constant considerably by choosing host matrices like PbTe, SnTe or GeTe. Our investigations of $\mathrm{Yb}$ doped $\mathrm{PbTe}$ and $\mathrm{PbS}$ showed that $\mathrm{Yb}$ exists as a divalent ion in $\mathrm{PbTe}: \mathrm{Yb}$, while mixed valency of $\mathrm{Yb}$ is observed in $\mathrm{PbS}: \mathrm{Yb}$ [1]; therefore the valency of $\mathrm{Yb}$ in SnTe is still a question. In the present paper preliminary results of X-ray photoelectron spectroscopy (XPS) experiments on RE-doped SnTe crystals are presented.

Pure and RE-doped SnTe crystals were grown by the Bridgman method taking the constituent elements in the stoichiometric proportion. The Sm, Eu, and $\mathrm{Yb}$ contents were analyzed by the electron microprobe analysis and found to be equal to $1.5,2.0$, and 1.5 at.\%, respectively.

The XPS spectra were recorded using a Perkin-Elmer small-spot ESCA spectrometer equipped with an Al X-ray tube, a Rowland-circle monochromator and 
a hemispherical electron energy analyzer. The spectral resolution was $0.7 \mathrm{eV}$ for valence-band spectra. Samples were introduced into the preparation chamber with a vacuum better than $10^{-9}$ torr, cleaved, and then shifted to the measuring chamber with a vacuum better than $5 \times 10^{-10}$ torr. The $3 d$ and $4 d$ photoelectron spectra of $\mathrm{Sn}$ and $\mathrm{Te}$ were measured for all of the studied crystals, but special attention was paid to the valence-band spectra and the most intensive core-levels signals of the rare-earth ions: $3 d$ for $\mathrm{Sm}$ and $\mathrm{Eu}$, and $4 d$ for $\mathrm{Yb}$ (the $\mathrm{Yb} 3 d$ spectrum is beyond the energy range of the spectrometer). No carbon or oxygen contaminations, even at the end of the experimental cycles, were detected.

The core-level spectra of $\mathrm{Sn}$ and Te did not show noticeable changes with doping by the considered RE ions.

The normalized valence-band photoemission spectra of $\mathrm{SnTe}, \mathrm{SnTe}: \mathrm{Yb}$, and the difference of the two spectra are presented in Fig. 1. The number of counts depends on the acquisition time, the contents of the elements and the experimental conditions. During the experiments the intensity of the X-ray tube remained constant within the accuracy of the spectrometer, but the shape of the analysed surface is not the same for all the samples. For taking into consideration the unknown "topography factor" we measure the most intense Te signal $-3 d$. Te contents in all the samples is $50 \%$ and we choose the same acquisition time for $3 d$ spectra. The Te $3 d$ lines count ratio gives us the factor indicating the intensity of the photoelectron process. The normalization procedure takes the acquisition time of the valence band spectra and the Te $3 d$ signal ratio in $\mathrm{Sn} T$ Te and RE doped $\mathrm{SnTe}$ crystals. The $\mathrm{SnTe}$ valence-band spectrum recorded in this work is similar to

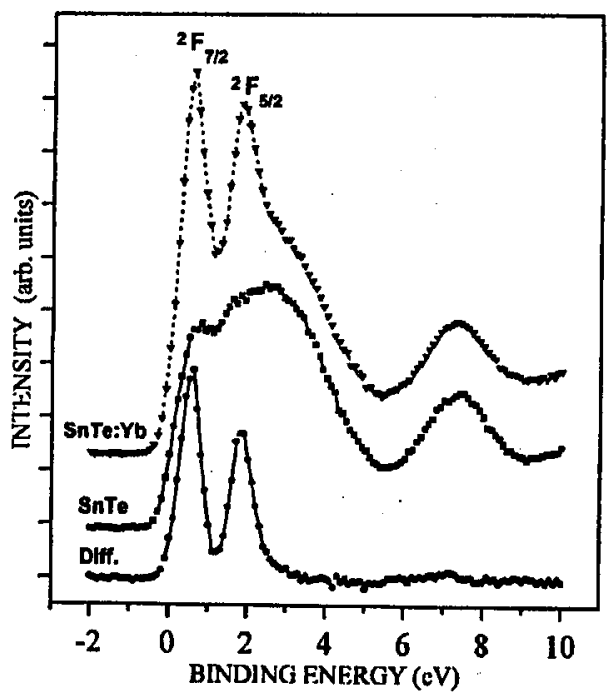

Fig. 1. XPS valence-band spectra of SnTe and SnTe:Yb. The bottom spectrum (filled circles and solid line) represents the difference of the two spectra after normalization (SnTe:Yb-SnTe). 
that reported earlier in the literature [2,3]. According to Kemeny and Cardona [3] the peak at $2.6 \mathrm{eV}$ originates from Te $5 p$ states with some admixture of Sn $5 p$ states. The peak centered at about $7.5 \mathrm{eV}$ is mostly due to $\mathrm{Sn} 5 s$ states with a strong admixture of $\operatorname{Sn} 5 p$ states. The difference between the valence-band spectra of SnTe and SnTe:Yb is most pronounced near the Fermi level. The shape of the valence electron energy distribution with two prominent peaks is characteristic of divalent $\mathrm{Yb}$ and arises from the $4 f^{14} \rightarrow 4 f^{13}$ photoionization process [4-6]. These two peaks can be assigned to ${ }^{2} F_{7 / 2}$ and ${ }^{2} F_{5 / 2}$ final states. The difference of the $\mathrm{SnTe}: \mathrm{Yb}$ and SnTe spectra can be fitted by the two Gaussian peaks with the following parameters: ${ }^{2} F_{7 / 2}$ state - energy at $0.53 \mathrm{eV}$ and total width (FWHM) of $0.55 \mathrm{eV} ;{ }^{2} F_{5 / 2}$ state $-1.85 \mathrm{eV}$ and $0.61 \mathrm{eV}$, respectively. The spin-orbit splitting of the doublet is found to be $1.32 \mathrm{eV}$, the area ratio is 1.29 and amplitude ratio is 1.44. The spin-orbit spliting of the $4 f$ electrons in divalent $\mathrm{Yb}$ metal is $1.27 \mathrm{eV}$ [4].

Figure 2 presents the normalized valence-band spectra for SnTe and SnTe:Eu and the difference of the two spectra. The modification induced by $\mathrm{Eu}$ ions is again located near the Fermi level. The maximum, centered at $1.4 \mathrm{eV}$, can be attributed to the ${ }^{7} F_{J}$ multiplet characteristic of $\mathrm{Eu}^{2+}$. The structure extends up to about $2.5 \mathrm{eV}$. A similar shape and position of the ${ }^{7} F_{J}$ multiplet has been found in $\mathrm{PbTe}: \mathrm{Eu}$, where $\mathrm{Eu}$ is also divalent [7].

A pronounced modification of the valence-band spectra introduced by $\mathrm{Sm}$ ions is found in the energy range from $5-10 \mathrm{eV}$ (see Fig. 3). The observed structure is characteristic of trivalent $\mathrm{Sm}$ ions and corresponds to the $4 f^{5} \rightarrow 4 f^{4}$ photoionization process $[8,9]$. The two broad structures located at $6.5 \mathrm{eV}$ and $9.0 \mathrm{eV}$ correspond to ${ }^{5} I$ and ${ }^{5} G$ final states. A more convincing proof for the trivalency of $\mathrm{Sm}$ in $\mathrm{SnTe}: \mathrm{Sm}$ is given by the $\mathrm{Sm} 3 d$ spectrum (not shown here).

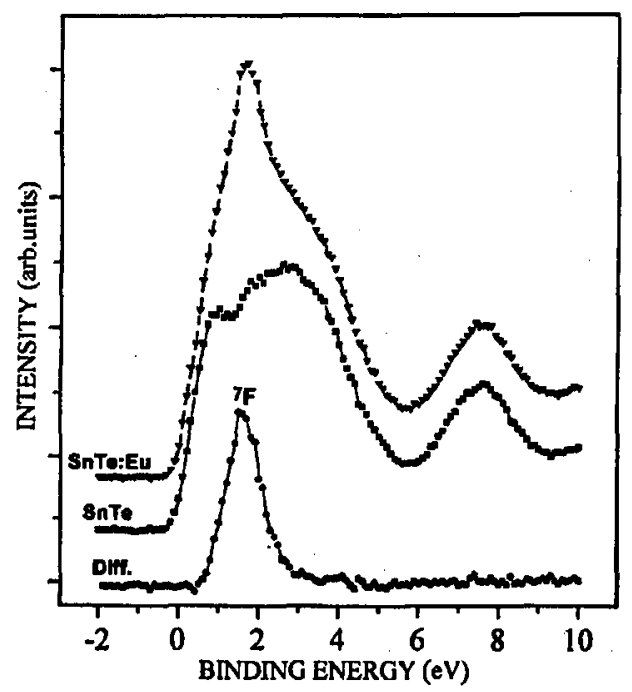

Fig. 2. XPS valence-band spectra of SnTe and SnTe:Eu. The bottom spectrum represents the difference of the two spectra after normalization (SnTe:Eu-SnTe). 


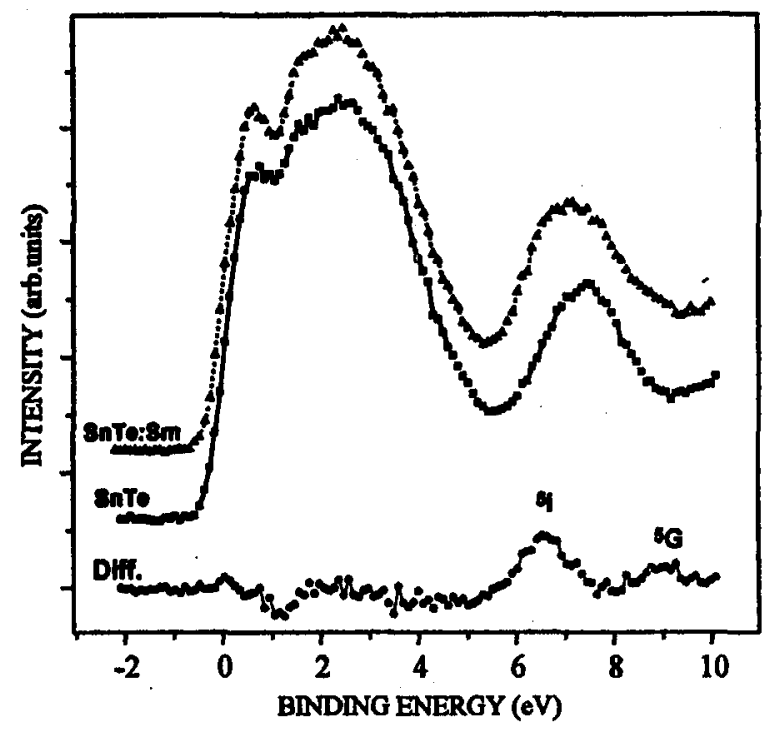

Fig. 3. XPS valence-band spectra of SnTe and SnTe:Sm. The bottom spectrum represents the difference between the two spectra after normalization (SnTe:Sm-SnTe).

The conclusions concerning the valency of $\mathrm{Eu}$ and $\mathrm{Yb}$ have also been confirmed by the analysis of RE core-level spectra not presented here ( $3 d$ for Eu and $4 d$ for $\mathrm{Yb}$ ).

In conclusion, we found that the valencies of rare-earth ions in the $\mathrm{SnTe}$ matrix can be different for different elements: $\mathrm{Sm}$ is trivalent while $\mathrm{Eu}$ and $\mathrm{Yb}$ are divalent. Doping of SnTe by these rare-earth ions in the range of a few atomic percent did not introduce noticeable changes in the core-level spectra of the matrix atoms (shape and energy position).

\section{References}

[1] Z. Gołacki, M. Heinonen, Acta Phys. Pol. A 91, (1997).

[2] I. Abbati, L. Braicovich, B. De Michelis, J. Phys. C, Solid State Phys. 7, 3661 (1974).

[3] P.C. Kemeny, M. Cardona, J. Phys. C, Solid State Phys. 9, 1361 (1976).

[4] K.H.J. Buschow, M. Campagna, G.K. Wertheim, Solid State Commun. 24, 253 (1977).

[5] R. Nyholm, Ib. Chorkendorff, J. Schmidt-May, Surf. Sci. 143, 177 (1984).

[6] W.F. Egelhoff, Jr., G.G. Tibbetts, Phys. Rev. Lett. 44, 482 (1980).

[7] M. Iida, T. Shimizu, H. Enomoto, H. Ozaki, Jpn. J. Appl. Phys. 32, 4449 (1993).

[8] F. Gerken, J. Phys. F, Met. Phys. 13, 703 (1983).

[9] Y. Baer, G. Busch, J. Electron Spectrosc. 5, 611 (1974). 